\title{
Organisational Cost and Complexity Saving Opportunities via the Development, Deployment, and Implementation of Blockchain Networks
}

\author{
Richard Bassett ${ }^{1}$, Mark Pisano ${ }^{2 *}$ \\ ${ }^{1,2}$ Southern Connecticut State University, Connecticut, USA
}

\begin{abstract}
Blockchain is being promoted as the platform to disrupt business as usual in many transaction-heavy sectors. Questions remain about the future standards of blockchain, their feature set, functionality, and the willingness of organisations to disrupt their existing revenue streams via blockchain. The most significant near-term promise that affords industry is the cost and complexity savings via the standardization of their technology infrastructure stacks. This paper explores the benefits available of reduced costs and complexity via the adoption of blockchain.
\end{abstract}

Keywords - Blockchain, banking, deployment, industries, human capital.

\section{INTRODUCTION}

Since the development of Bitcon and its underlying technology of blockchain in 2008 by Satoshi Nakamoto [1], there has been many questions as to the direction of the development of the technology and more importantly how well received or adopted it will become across industries. Financial segments saw early success with crypto currency with Bitcoin leading the development and consumer adoption. It was and remains a very popular currency exchange among consumers.

Outside of crypto currency, blockchain technology has much more to offer the world, and organisations from all types of industries have taken notice and have begun to invest or look into the potential uses that blockchain can have for them. Adoption, development, and implementation of blockchain networks will have costs associated with them but the long-term goal is to see cost savings from the investment. Secondarily, organisations maybe spending on the evaluation of blockchain technologies due to fear that they may be left behind in their industry while their competition reaps rewards from being an early adopter of a technology that is being heavily discussed.

This paper reviews the underlying distribute ledger technology, the impact that it has on not just the financial and technology industries but other industries that have begun to show interest along with discussion of the current state of blockchain and the near-term opportunities that can be gained from using a blockchain network.

\section{BEYOND BITCOIN - DisTRIBUTED LEDGER TECHNOLOGY}

Bitcoin, which is built on blockchain technology, has been a presence since 2008 when it was first introduced in a whitepaper entitled Bitcoin: A Peer-to-Peer Electronic Cash System by Satoshi Nakamoto [2]. Bitcoin was the predominant application of blockchain until 2013 when technology entrepreneurs realised that blockchain has considerably more potential applications beyond cryptocurrency and started envisioning the applicability of extending blockchain to other business applications.

Blockchain, an open, decentralized ledger that records transactions between multiple parties in a permanent, immutable way without the need for third-party gatekeepers, is the most widely known distributed ledger technology (DLT). A decentralized distributed ledger is a consensually shared and synchronized database that exists across multiple network nodes, participants, sites, institutions or geographies eliminating the need for a central authority or an intermediary to process, validate or authenticate transactions [3]. DLT allows for storage of information in a secure and consistent fashion using cryptography which can be accessed using cryptographic signatures and keys. Stored information becomes part of an immutable database that is governed by the rules of the network stored in [4].

Some of the return on investment (ROI) benefits of leveraging distributed ledger technology include time savings, cost savings, elimination of single-point-of-failure risks associated with centralized databases, reducing operational inefficiencies, increased security through cryptography, immutability and the potential elimination of 3rd party intermediaries used to process, validate or authenticate transactions or other types of data exchanges.

A properly executed DLT removes the need for users of a system to trust a third party or each other as long as records that are stored in the ledger are done so only when consensus has been reached by the authorized parties involved. Blockchain consensus mechanisms include: Proof of Work (PoW), Proof of Stake (PoS), Delegated Proof of Stake (DPoS), Proof of Authority \& Proof of Importance [5].

\footnotetext{
${ }^{*}$ Corresponding author. E-mail: pisanom1@southernct.edu
} 
Traditionally, most sizeable corporate enterprise resource databases are centralized and under the control and governance of their corporate ownership. Trusted corporate employees typically decide on how the contained data are stored, altered, and disseminated. Users of such systems are forced to trust the companies that control them. Whereas, a decentralized distributed ledger system removes the necessity of a single organisation managing the data, which is why they are often described as 'trustless' [6].

\section{DLT DEPLOYMENT IN VARIOUS INDUSTRY SECTORS AND THE IMPACT OF COVID-19}

Numerous large corporations, joint ventures and government entities such as Microsoft, BHP BILLITON, Maersk, BITA ALLIANCE, United Nations, Electron/Tepco, and Illinois IDFPR and many more are currently working on various DLT applications [7].

As of 2019, DLT was being deployed in the following industries: Banking and Finance, International Trade and Commodities, Supply Chain, Energy, Identity, Real Estate, Government \& Public Records, Healthcare, Media and Entertainment and Sports, Business Technology [8].

In addition to the sectors identified above, DLT applications were being explored and developed in the following industry areas: Messaging Apps, Voting, Infrastructure Security, Ride Sharing, Internet Advertising, Education \& Academia, Car Sales \& Leasing, Music \& Entertainment, Stock Trading, Insurance, Energy Management, Gift Cards \& Loyalty Programs, Gun Tracking, Legal, Retail, Charity, Law Enforcement, Human Resources, Corporate Governance, Credit Histories, Manufacturing, Printing, Libraries, Publishing, Fishing, Photography, Gaming, Food \& Beverage, Travel, Agriculture, Gambling, Pharma, Trucking, Waste Management, and Construction [9].

All of these industries and the businesses within them are looking to leverage the potential benefits that come with DLT. This ranges greatly by industry and business but the promise of long-term reduced costs, greater efficiency, and endless automation is very attractive. In addition to the potential shortterm benefits, the idea of being left behind or losing out to a competitive advantage helps drive the decision-making process.

As investment interest in DLT was starting to gain momentum heading into 2020, then the COVID-19 global pandemic hit, catching the business world totally off-guard and ill-prepared to conduct business as usual let alone to afford the luxury of developing new technologies. Organisations had to focus on the minimal things necessary to 'keep the lights on' in their businesses and put growth plans and opportunities such as DLT on hold while they scrambled in survival mode.

The still unfolding COVID-19 pandemic is anticipated to have a considerable impact on the growth of blockchain distributed ledger industry [10]. The pandemic illuminated many shortcomings in existing business processes and the underlying technologies that support them, especially those involving worker's ability to perform their jobs remotely. Most organisations were ill-prepared for a small portion of their workforce to be working remotely yet alone their entire organisations to be off-site with virtually no warning. This sudden shift forced organisations to quickly divert IT operations and to adopt technologies and new business processes to facilitate an entirely remote workforce.

Some organisations have managed to leverage the challenges of the pandemic as a catalyst for rapid DLT deployment, including several British hospitals in the NHS that are using blockchain in the vaccine rollout [11]. The hospitals are using DLT to monitor the storage and distribution of COVID-19 vaccines, alongside chemotherapy drugs and other vaccines [12].

The pandemic has also led to consideration of other practical DLT use cases to be considered, including contact tracing, tracking medical supplies, patient record sharing, and provider credentialing while the healthcare industry responds to the demands of the pandemic [13].

While many organisations have already begun developing and deploying distributed ledger solutions, it is unclear how much success that they were having as blockchain application deployment, beyond cryptocurrency, is still at its infancy stages, primarily due to evolving standards.

\section{BANKING/FINANCE AND TECHNOLOGY SECTOR INVESTING HEAVILY}

The sectors with the most substantial investment in DLT are banking, Fintech, and technology companies as they stand the most to gain on the upside success of the widespread adoption of DLT and stand the most to lose by maintaining the status quo if blockchain and DLT displace existing business technology going forward.

Traditional major banks are investing heavily in blockchain to preserve their existing revenue streams in the face of the challenges being put forth by a legion of Fintech start-ups to their existing business models. For every service offered by traditional major banks, there is at least one Fintech start-up offering similar deals at a lower cost and increased convenience [14].

"Blockchain is a disruptive development and banks have a lot of fear concerning this technology because, in the pure theory of blockchain, a lot of processes within a traditional bank would be obsolete," says former Citibank chief executive John Reed.

A clear example of the disruptions possible to the overall finance industry deals with the processing of credit card transactions or the transfer and movement of money. Traditionally, as consumers use credit cards or transfer funds around, the financial organisations take percentage of the transactions along with fees to profit and maintain the financial network of processing gateways. The development and use of crypto currency has eliminated a large portion of the costs associated with these types of transactions. Consumers have been able to move and spend crypto currency without having to deal with the centralized financial network.

Blockchain is a core technology in Fintech. The original design of blockchain focused on the cryptocurrency "Bitcoin". Fintech stands to disrupt every business client sector that the traditional banking and financial industry service. 
Besides the substantial investments in blockchain by the banking and Fintech, the technology solution provider sector is also making substantial investments in the future of blockchain and DLT. This is evidenced by their hiring, investments, and the strategic industry partnerships that they have formed. One such example of strategic partnerships is between DLT Solutions and cloud provider Amazon Web Services (AWS) that has been named a Public Sector Distribution Partner for AWS [15].

Technology Solution Providers provide infrastructure, cloud services, software, consulting services, best practices in business processes, and the tools used by enterprise computing users across all industry sectors. In short, they provide the underlying technology solutions needed to make DLT and blockchain work. Like Fintech start-ups, they cross into all client business sectors and are the 'tech' component of Fintech.

Blockchain and DLT business represent a potential financial bonanza on the scale of the initial internet boom of the late 1990s for technology service providers. Most existing ERP companies, consulting firms, and system integrators are organised around industry sectors. Technology solution providers and Fintechs aim to span across the traditional client model of industry sectors.

Corporate clients tend to stay with their enterprise technology providers as they are often locked in as the cost and effort and risk of switching to another provider are too great. Small incremental improvements, enhanced technology features, are not generally compelling enough to entice an enterprise client to switch from one technology platform to another but a total make-over of business applications and the platforms which they run on may offer a more compelling case in the face of quantum benefits and cost savings, competitive advantage and if their trusted advisors endorse such changes.

The investment, hiring, publicity, and excitement in the DLT space create a substantial opportunity for technology solution providers to essentially hit the reset button on their corporate offerings, the opportunity to shuffle the deck chairs on their customer base.

\section{BLOCKCHAIN AND DLT HIRING - CURRENT INVESTMENTS IN HUMAN CAPITAL}

A quick search of a few of the most popular job sites, LinkedIn and Indeed, have been returning 3500-5000 blockchain positions inclusive of cryptocurrency. The majority of these positions are in Information Technology and Services (primarily software development), Financial Services, Management Consulting followed by infrastructure.

Most of the recruiting taking place appears to be by providers of technology and consulting services, major banks and financial institutions or start-ups developing solutions for them, very little blockchain recruiting seems to be happening at direct client end-user companies outside these sectors. The pandemic could create significant hiring opportunities in health and medical fields, as many organisations have implemented blockchain and DLT solutions to help support medical record data and issues around the distribution and tracking of vaccines.
The hiring data would suggest that the technology sector is trying to get a jump on the business application deployment and related services of blockchain to drive the change to their current and future customer base. This can also signify that there has been interest expressed by current and potential customers for blockchain offerings and the related services. These customers are most likely investigating how blockchain and blockchain services could benefit them driving the technology sector to scale up in anticipation of the future sales and service work. It would also further imply that the banking industry recognises the risks and opportunities that blockchain presents to their existing business models and is investing substantially in human capital. It may be their hope to scale up their use of blockchain and its services to further push a direction for standardized use allowing them to shape the acceptance and direction of blockchain as a whole.

\section{DIFFERENT TYPES OF DLT, COMPETING DLT PLATFORMS AND EVOLVING STANDARDS}

There are many different types of DLTs, including Blockchain, Hashgraph, DAG, Holochain, Tempo [16] each with unique feature sets and different methods of operation from each other offered by competing platforms. See Table I for current most notable platforms provided.

TABLE I

CURRENT OFFERINGS OF BLOCKCHAIN AND DISTRIBUTED LEDGERS

\begin{tabular}{|l|l|l|l|l|}
\hline Blockchain & Hashgraph & DAG & Holochain & Tempo \\
\hline Ethereum & Hedera & IOTA & Holochain & Radix \\
$\begin{array}{l}\text { Hyperledger } \\
\text { Fabric }\end{array}$ & Hashgraph & Byteball & & \\
c.rda & NOIA & & & \\
\hline
\end{tabular}

Some platforms are fully developed, while others are still emerging. Some strictly adhere to the blockchain standard and others have built upon it and thus deviate from the blockchain standard. Several platforms employ distributed ledgers without the use of blockchain. These blockchain network types can be defined as public, private, consortium, federated, hybrid, or semi-private [17].

The challenge for all stakeholders seeking to invest, develop, and build-out DLTs is that standards are still rapidly evolving, DLTs and their respective feature sets from one platform are not necessarily compatible with others.

The DLT platforms continually advance their products by creating new revisions or versions to the chain. They look to expand the size of storage, speed up process times, enhance security, and add new features. This is a continuous cycle similar to the software development cycle and adds additional confusion as to what solution would truly be best for the end user organisation or what would be best suited to meet the technology problem at hand. An example of a major change or enhancement to a blockchain or DLT platform would be the addition of the smart contract.

This vast number of DLT offerings combined with their array of differences makes it very difficult for organisations to have a clear picture of what solution to implement or which one could arise as the standard. To further add to the confusion, 
without a clear standard, organisations run the risk of not being able to integrate with partners if they have adopted a different offering.

If DLTs are going to achieve global interoperability and acceptance, there will most likely be consolidation and standardization making winners and losers amongst the competing platforms and crushing the efforts and investments of early adopters who hitched their wagon to an obsolete standard or platform. A wide variety of platform choices make it difficult and risky for businesses to invest without having a sense as to what the dominant platform(s) will be.

\section{CURRENT STATE AND NEAR-TERM OPPORTUNITIES}

The success of Bitcoin and the widespread publicity of other cryptocurrencies have propelled blockchain and distributed ledger technology onto the radar of major banks, Fintech companies, governments, technology companies, consulting firms, venture capitalists and direct client companies as the promising technology exhibits the potential to disrupt business as usual with quicker transaction processing time, reduced intermediary fees, better-organised records, secure records, and highly advanced workflows.

Much like early mainframe computers and personal computers blockchain has often been described as a solution in search of a problem, but as investment steadily increases in the application technology, blockchain and DLTs are moving out of the start-ups and research phase into some deployable enterprise applications.

Until blockchain and DLT standards evolve into a standard, or two, it is difficult and risky for technology companies to fully invest in and build out their full suite of enterprise client-centric offerings of product and services. It is equally risky for enterprise clients to build out specific blockchain or DLT solutions in the absence of clear standards.

In response to the threats posed by entrepreneurial Fintechs, major banks have recognised the profitability risk of sticking with the status quo business models and are already investing heavily in blockchain, cryptocurrencies, and DLTs. A large percentage of the blockchain-related hiring that has been taking place for the past 12 months has been by major banks and financial institutions.

Banks and major financial institutions are already preemptively rolling out cloud-based blockchain and DLT solutions to their clients, while they are still at their infancy stages, these trusted institutions have considerable clout with their existing customer base. Cloud service companies are incorporating DLT in their arsenal of offerings, which are being leveraged by banks and major financial institutions. Interestingly enough, the largest blockchain patent holder is Bank of America [18].

Before the global pandemic, major industry oversight bodies began to utilize blockchain technology to leverage the security and data efficiencies. The United States Food and Drug Administration (FDA) has been utilising a blockchain pilot program, which is intended to help streamline the auditing of medication. The FDA hopes that using blockchain will significantly reduce the time required to audit medication, increase its accuracy, enhance their tracking capabilities, and create more efficiency. This will in turn allow for more types of medication to be tracked promoting a safer drug supply chain [19]. In addition to the FDA, other major United States government agencies are investing in or piloting the use of blockchain. This includes the United States Department of Health and Human Services (HHS) and the Unites States Center for Disease Control (CDC) [20]. If any of these major industry oversight bodies deem their pilot programs viable, it could be the catalyst that pushes blockchain out of the financial industry and to a position of mainstream use across vast supply chains. There would most likely be a cascading effect as manufacturing and supply companies look to meet new technical requirements to meet the governing demands and push their partners to adopt similar requirements.

As the world evolves out of the global pandemic, lessons learned in regards to the use of blockchain and DLT for medical record storage, vaccine tracking, and the use of medical or vaccine passports will be evaluated. If deemed successful, with such a large and vigorous test pool, the medical industry could see large scale adoption and implementation of blockchain and DLT across all of its data domains to leverage cost savings, reduced operational inefficiencies, removal of single points of failure, increased security, and the potential mandates required by government oversight bodies.

\section{CONCLUSION}

Bitcoin and cryptocurrencies have created tremendous interest in blockchain and its derivative technologies. Some organisations are claiming early success with blockchain and distributed ledgers enabled applications. However, it is unclear how the global pandemic has disrupted the investment and development of blockchain technologies. Research and markets expect investments in these technologies to rise from $\$ 1.2$ billion in 2018 to more than $\$ 23.3$ billion by 2023 [21]. Medical, banking, Fintech, and technology solution providers are best positioned to be the early beneficiaries of blockchain and DLTs should they become commercially successful.

Considerable obstacles are standing in the way of blockchain and DLTs of becoming successful [22] with the largest being the fragmentation of industry standards and competing platforms, as well as the willingness of client enterprise organisations to replace their existing expensive technology infrastructures with blockchain-enabled technology. To get enterprises to move from their current-state single-instance centralized environment to a blockchain distributed environment will require substantial investments in projects, time, and resources. Technology solution providers will have to be able to demonstrate that they have proven end-to-end applications for those type of wholesale changes in corporate infrastructures.

The fear of being left behind by industry competitors who have initial success in the early adoption of blockchain and DLTs is a real concern to many corporate CEOs, and the risk to having their business models upended by some of the promised benefits of smart contracts and public ledgers cannot be easily ignored. However, the initial costs, requirements, and potential 
disruption to processes could be overbearing leaving many organisations and industries sitting on the side lines taking a slow and conservative approach to the evaluation and ultimate adoption of blockchains and DLTs.

In the near-term direct-client enterprises can protect themselves by getting ready for the possibility of pervasive blockchain and DLT success by investing in education for critical members of their organisation relative to its opportunities and risks, by making strategic preliminary investments in new personnel, implementing critical dualpurpose replacement infrastructure, and by entering into partnerships with cutting edge industry leaders in blockchain technology. This way, an organisation could be ready to quickly and efficiently scale a DLT technology in a shorter time frame and allow for a potential standardized solution to become available. It also has the advantage of reducing the overall risk that the organisation would be in a position of being left behind if competitors implement solutions before them.

Executives and leaders need to appreciate how blockchain and DLT may or may not impact their organisations, investments in their knowledge in this area will be critical. Key strategic planners must understand how their current and forecasted future business models map to the industry solutions that banks, Fintechs, and technology solution providers are actively working on, as well as an understanding of the possible strategic partnerships with other key stakeholders for public and semi-private ledgers. It is likely that existing banking and technology vendors of direct-client companies are already making investments in blockchain-enabled products and services and have some available. Clients may consider evaluating or adopting some of these early offerings as a way to experience and learn more about the practical aspects of the technology.

If banks, Fintechs, and technology solution providers work out all of the major obstacles of blockchain and DLTs and develop comprehensive cross-industry solutions, then directclient companies can anticipate that a forced migration to the new platforms will be forthcoming as old technologies will be discontinued and rendered unsupported.

Additionally, it is unclear as to the effect that the global pandemic has had on the development of blockchain and DLTs. Some movement has been made in the medical industry to use blockchain and DLTs to help facilitate medical records and vaccines. With the success of these endeavours it should be expected that the medical industry will start to see a large-scale adoption of blockchain and DLT solutions, potentially putting their use to the same utilization and acceptance as the financial industry.

It is becoming clear that with all of the discussion and interest in blockchain and DLT, more and more solutions will be forth coming and their use across multiple industries will continue to grow bringing an early technology to a mature, well rounded offering that could be streamlined to support all types of industries and meet the needs of complex information technology problems.

Blockchain and DLT as a whole have moved out of being just a supporting tool to crypto currency. As there are many different offerings and more being developed, the competition between platforms has spawned newer, faster, and more comprehensive versions. This in part does add to some confusion amongst standardization but it clearly shows that the technology is on its path to maturity and it will most likely reach a position of universal interoperability and adoption by a large number of industries.

\section{REFERENCES}

[1] S. Nakamoto, "Bitcoin: A peer-to-peer electronic cash system," Oct. 2008. [Online]. Available: https://bitcoin.org/bitcoin.pdf. Accessed on: May 28, 2020

[2] M. Isansiti and K. Lakhani, "The truth about blockchain," Jan. 2017. [Online]. Available: https://enterprisersproject.com/sites/default/files/the_truth_about_blockc hain.pdf. Accessed on May 28, 2020.

[3] A. Meloa, "How distributed ledger technology will change the way the world works," Oct. 5, 2017. [Online]. Available:

https://www.businessinsider.com/blockchain-distributed-ledgers-201710. Accessed on: May 28, 2020.

[4] J. Frankenfield and E. Rasure, "Distributed ledger technology," Mar. 31, 2021. [Online]. Available:

https://www.investopedia.com/terms/d/distributed-ledger-technologydlt.asp Accessed on: July 4, 2021.

[5] A. Stevens, "Distributed ledger consensus explained," Apr. 29, 2018. [Online]. Available: https://hackernoon.com/distributed-ledgerconsensus-explained-b0968d1ba087. Accessed on: May 28, 2020.

[6] D. Yafimava, "Consensus in blockchain: What you need to know," Aug. 9, 2019. [Online]. Available: https://openledger.info/insights/blockchainsmart-cities. Accessed on: May 28, 2020.

[7] "80+ corporations working on blockchain and distributed ledgers," CBInsights, Jan. 3, 2019. [Online]. Available: https:/www.cbinsights.com/research/organizations-corporates-testblockchains-distributed-ledgers. Accessed on: May 28, 2020.

[8] "Blockchain use cases and applications by industry," Consensys, 2019. [Online]. Available: https://consensys.net/enterprise-ethereum/use-cases. Accessed on: May 28, 2020.

[9] "Banking is only the beginning: 55 big industries blockchain could transform," CBInsights, June 11, 2011. [Online]. Available: https://www.cbinsights.com/research/industries-disrupted-blockchain. Accessed on: May 28, 2020.

[10] P. Borasi and V. Modi, "Allied market research blockchain distributed ledger market," [Online]. Available: https://www.alliedmarketresearch.com/blockchain-distributed-ledgermarket. Accessed on: July 5, 2021.

[11] J. Denton, "Crypto tech vs. COVID-19: How hospitals are using blockchain in the vaccine rollout," Jan. 20, 2021. [Online]. Available: https://www.marketwatch.com/story/crypto-tech-vs-covid-19-howhospitals-are-using-blockchain-in-the-vaccine-rollout-11611079259. Accessed on: July 5, 2021.

[12] K. Afifi, "NHS turns to distributed ledger tech to manage COVID-19 vaccine rollout," Jan. 20, 2021. [Online]. Available: https:/www.itpro.com/technology/358377/nhs-using-dlt-to-monitorcovid-19-vaccines. Accessed on: July 5, 2021.

[13] B. Furst, "How COVID-19 has revealed healthcare's blockchain use cases," Nov. 17, 2020. [Online]. Available: https://www.hitconsultant.net/2020/11/17/covid-19-healthcareblockchain-use-cases/\#.YOZRQxNKi3d. Accessed on: July 5, 2021

[14] "Banks and the FinTech challenge: How disruption has been a catalyst for collaboration and innovation," Currencycloud. [Online]. Available: https://mp.s81c.com/pwb-production/000001partner/7730/documentation/15704_en.pdf. Accessed on: May 28, 2020.

[15] "DLT solutions named Amazon Web services public sector distribution partner," DLT, Nov. 27, 2018. [Online]. Available: https://www.dlt.com/news/dlt-solutions-named-amazon-web-servicespublic-sector-distribution-partner. Accessed on: June 3, 2020.

[16] H. Anwar, "Distributed ledger technology: Where technological revolution starts, 101 blockchains," Jan. 30, 2019. [Online]. Available: https://101blockchains.com/distributed-ledger-technology-dlt. Accessed on: June 3, 2020

[17] "Blockchain platforms, networks and other distributed ledger technologies (DLT)," HIMS, 2019. [Online]. Available: 
https://www.himss.org/library/blockchain-platforms-networks-andother-distributed-ledger-technologies-dlt. Accessed on: June 3, 2020.

[18] H. Son, "Bank of America tech chief is skeptical on blockchain even though BofA has the most patents for it," $C N B C$, Mar. 26, 2019. [Online]. Available: $\quad$ https://www.cnbc.com/2019/03/25/bank-of-americaskeptical-on-blockchain-despite-having-most-patents.html. Accessed on: May 28, 2020.

[19] U.S. FDA, "FDA takes new steps to adopt more modern technologies for improving the security of the drug supply chain through innovations that improve tracking and tracing of medicines," Feb. 7, 2019. [Online] Available: https://www.fda.gov/news-events/press-announcements/fdatakes-new-steps-adopt-more-modern-technologies-improving-securitydrug-supply-chain-through. Accessed on: May 26, 2020.

[20] J. Calvin et al., "Blockchains for government: Use cases and challenges," Digital Government: Research and Practice, vol. 1, no. 3, art. 22, Nov. 2020. [Online]. Available: https://doi.org/10.1145/3427097. Accessed on: July 5, 2021.

[21] "Blockchain market by provider, application (payments, exchanges, smart contracts, documentation, digital identity, supply chain management, and GRC management), organization size, industry vertical, and region Global forecast to 2023," Research and Markets, Dec. 2018. [Online] Available: https://www.researchandmarkets.com/reports/4703993/blockchainmarket-by-provider-application. Accessed on: May 28, 2020.

[22] "Real-world uses of the blockchain today, from supply chain to equity," Computerworld, Sep. 4, 2019. [Online]. Available: https://www.computerworld.com/article/3430225/real-world-uses-ofthe-blockchain-today-from-supply-chain-to-equity.html. Accessed on: June 3, 2020 .
Richard Bassett earned his Doctorate in Computer Information Systems from Pace University in 2003, his Master degree in Computer Information Systems and his Bachelor degree in Computer Science from the University of New Haven. He is a tenured Associate Professor of Information Systems and Chair of the Business Information Systems Department at Southern Connecticut State University in New Haven, CT and was formerly the Assistant Dean of the Business School at Western Connecticut State University.

E-mail: bassettr3@southernct.edu

Mark Pisano is an Assistant Professor of Business Information Systems at Southern Connecticut State University. He received his Doctorate in Computing Studies from PACE University. His areas of interest are in computer security, digital forensics, virtualization, data centers, and enterprise computing networks. He has published in ACET Journal of Computer Education and Research.

E-mail: pisanom1@southernct.edu

ORCID iD: https://orcid.org/0000-0002-6268-0131 